\title{
Concept d'évaluation gériatrique. Applications à la médecine gériatrique en 2009
}

\author{
P. Chassagne \\ (C) Springer-Verlag France 2009
}

Le concept d'évaluation gériatrique globale (comprehensive geriatric assessment) est fondé sur des principes fondamentaux. Ces principes peuvent se résumer en trois points :

- la nécessité d'une approche globale médicopsychosociale du malade ou de la personne âgée ;

- l'hétérogénéité des sujets âgés qui se caractérise par des notions allant du vieillissement physiologique à la vulnérabilité, à la fragilité ou encore au vieillissement polypathologique ;

- les caractéristiques des personnes âgées qui, indépendamment de leur âge et de leur comorbidité (synonyme de polypathologie), souffrent aussi de syndromes gériatriques. Ces syndromes gériatriques tels que les chutes, l'incontinence urinaire ou encore l'existence d'un syndrome confusionnel sont souvent multiples. Ils peuvent être intriqués et, s'ils sont négligés, avoir des conséquences délétères sur la santé des sujets âgés.

Les besoins sanitaires et sociaux des personnes âgées ne peuvent donc être uniquement fondés sur la prise en compte, isolément, de leurs multiples pathologies. Ces besoins justifient une évaluation multidimensionnelle et multiprofessionnelle dont les premières applications sont intervenues entre 1984 et 1995, avec les travaux de Rubenstein et al. et de Stuck et al. notamment $[1,2]$.

Dans un premier temps, les auteurs se sont attachés à valider ce modèle d'évaluation multidimensionnelle qui avait pour objectif d'appréhender, simultanément, les caractéristiques du malade âgé, de sa maladie (exemple : spécificité sémiologique ou thérapeutique liée à l'âge) et de son entourage. Ces études ont permis de souligner l'intérêt de cette approche, par exemple sur la survie des sujets pris en charge ou sur la préservation de leur autonomie fonctionnelle. L'évaluation gériatrique globale fait désormais partie des pratiques de routine des équipes gériatriques qui, après

\footnotetext{
P. Chassagne $(\square)$

Service de médecine interne gériatrique,

CHU de Rouen, 1, rue de Germont,

F-76031 Rouen cedex, France

e-mail : philippe.chassagne@chu-rouen.fr
}

s'être formées, ont appris à manier quotidiennement les outils nécessaires à sa réalisation.

La spécificité de l'évaluation gériatrique globale, outre sa méthode proprement dite, repose sur la notion d'intervention d'une équipe pluriprofessionnelle. La composition de cette équipe varie selon les centres où l'évaluation gériatrique intervient. Elle est composée, au minimum, d'un médecin gériatre, d'une infirmière spécialisée, d'un secrétariat, d'une assistante sociale. D'autres professionnels, comme les ergothérapeutes, les spécialistes en nutrition ou bien encore les psychomotriciens, complètent, selon les cas, ces équipes.

Depuis dix ans maintenant, l'évaluation gériatrique globale est au cœur des pratiques médicales des équipes gériatriques qui interviennent au service d'accueil et d'urgences, dans les milieux d'oncologie, de néphrologie ou encore dans les services de chirurgie. Pour ces différents spécialistes, l'évaluation globale gériatrique trouve sa place dans la prise de décision thérapeutique initiale (telle la décision de réaliser une chimiothérapie chez un malade âgé traité pour un cancer qui fait l'objet d'un article détaillé dans ce numéro spécial) ou bien encore pour optimiser la prise en charge d'une fracture de l'extrémité supérieure du fémur. La fracture de l'extrémité supérieure du fémur est une maladie gériatrique dont les conséquences sont redoutables en termes de morbimortalité. Ces conséquences sont atténuées par une prise en charge partagée, entre le chirurgien, l'anesthésiste ou le gériatre, de la polypathologie mais aussi des syndromes gériatriques du sujet âgé.

Pour les gériatres, à proprement parler, l'évaluation globale gériatrique fait partie des soins, qu'ils se dispensent en ambulatoire (évaluation gériatrique globale dans le cas des activités des unités mobiles gériatriques, des consultations d'évaluation gérontologique), en milieu hospitalier ou dans les institutions. Dans les institutions, l'évaluation gériatrique globale permet de mieux définir le plan de soins en le personnalisant à chaque résident âgé.

Dans tous les cas de figure, quelles que soient les conditions de vie du malade âgé, l'évaluation gériatrique globale n'a de sens que si elle aboutit à formuler un plan de soins 
personnalisé. Le plan de soins personnalisé a trois objectifs majeurs pour chaque personne âgée prise en charge : l'amélioration de sa survie, la préservation de son autonomie fonctionnelle et de sa qualité de vie. Ce plan de soins, souvent initié en milieu hospitalier, permet, en collaboration étroite avec le médecin généraliste, de garantir une meilleure prise en charge des sujets âgés.

\section{Références}

1. Rubenstein LZ, Josephson KR, Wieland GD, et al (1984) Effectiveness of a geriatric evaluation unit: a randomised clinical trial. New Engl J Med 311:1664-70

2. Stuck AK, Aronow HU, Steider A, et al (1995) A trial of annual in-home comprehensive geriatric assessment from elderly people living in the community. New Engl J Med 333:1821-29 